\title{
ESTIMATING AND COMPARING CANCER PROGRESSION RISKS UNDER VARYING SURVEILLANCE PROTOCOLS ${ }^{1}$
}

\author{
By Jane M. Lange*,1,2, Roman Gulati*,1,2 , Amy S. LeOnARdson*,1,3, \\ DANIEL W. LiN ${ }^{\dagger}, 1,4$, LiSA F. NEWCOMB ${ }^{*, 1,4}$, BRUCE J. TROCK ${ }^{\ddagger}, 1$, \\ H. Ballentine Carter ${ }^{\ddagger}, 1$, Peter R. CARroll ${ }^{\S, 1,5}$, \\ MATTHEW R. COOPERBERG ${ }^{\S}, 1,5$, JANET E. COWAN ${ }^{\S}, 1,5$, \\ LAWRENCE H. KLOTZ ${ }^{\text {II, } 6}$ AND RUTh ETZIOnI ${ }^{*} \dagger, 1,2$ \\ Fred Hutchinson Cancer Research Center*, University of Washington ${ }^{\dagger}$, \\ Johns Hopkins University ${ }^{\ddagger}$,University of California, San Francisco ${ }^{\S}$ \\ and University of Toronto ${ }^{\text {II }}$
}

\begin{abstract}
Outcomes after cancer diagnosis and treatment are often observed at discrete times via doctor-patient encounters or specialized diagnostic examinations. Despite their ubiquity as endpoints in cancer studies, such outcomes pose challenges for analysis. In particular, comparisons between studies or patient populations with different surveillance schema may be confounded by differences in visit frequencies. We present a statistical framework based on multistate and hidden Markov models that represents events on a continuous time scale given data with discrete observation times. To demonstrate this framework, we consider the problem of comparing risks of prostate cancer progression across multiple active surveillance cohorts with different surveillance frequencies. We show that the different surveillance schedules partially explain observed differences in the progression risks between cohorts. Our application permits the conclusion that differences in underlying cancer progression risks across cohorts persist after accounting for different surveillance frequencies.
\end{abstract}

1. Introduction. Many outcomes after cancer diagnosis and treatment are observed at discrete times via doctor-patient encounters or specialized diagnostic examinations [Sridhara, Mandrekar and Dodd (2013)]. For example, prostate cancer

Received May 2017; revised September 2017.

${ }^{1}$ Supported by the National Cancer Institute Award Number R01 CA183570 for the Prostate Modeling to Identify Surveillance Strategies (PROMISS) consortium.

${ }^{2}$ Supported by the National Cancer Institute Award Number U01 CA199338 as part of the Cancer Intervention and Surveillance Modeling Network (CISNET).

${ }^{3}$ Supported by the National Cancer Institute Award Number P50 CA097186 as part of the Pacific Northwest Prostate Cancer Specialized Program in Research Excellence (SPORE).

${ }^{4}$ Supported by the Canary Foundation.

${ }^{5}$ Supported by Genomic Health Inc. and the US Department of Defense Translational Impact Award for Prostate Cancer Award Number W81XWH-13-2-0074.

${ }^{6}$ Supported by Prostate Cancer Canada.

Key words and phrases. Hidden Markov model, multistate model, panel data, prostate cancer, active surveillance. 
progression following primary surgery is typically asymptomatic and is identified by a high or rising prostate-specific antigen (PSA) level on follow-up testing [Stephenson et al. (2006)]. Similarly, breast cancer recurrence after an initial diagnosis of in-situ disease is generally identified by surveillance mammography screening [Narod and Rakovitch (2014)]. A surveillance-dependent random variable is a continuous-time failure outcome that is detected by exams or biomarker measurements that occur at discrete times (e.g., patient visits). A key feature of studies with surveillance-dependent outcomes is that the observed time of an event is sensitive to the frequency of patient visits: patients with more frequent visits will have an event detected earlier [Gignac et al. (2008), Zeng et al. (2015)]. Other differences across surveillance studies include inconsistent definitions of failure and variable frequencies of dropout, each of which may affect estimated risks of the event.

In this article, we consider the problem of comparing risks of cancer progression across multiple cohorts with different surveillance frequencies, where inconsistent definitions of progression were involved and variable frequencies of dropout were observed. We present a statistical framework based on multistate models [Andersen and Keiding (2002)] that considers a standardized definition of cancer progression as an event that occurs on a continuous time scale and accounts for dependent censoring due to the variable dropout.

Our application focuses on the setting of prostate cancer grade progression among patients on active surveillance. In active surveillance studies, patients with low-risk prostate cancer do not undergo active treatment at the time of diagnosis but rather are assigned to a schedule of regular biopsies and PSA measurements to monitor disease progression. Patients are generally referred to treatment if progression is detected, but they may also initiate treatment at any time for other reasons, including rising PSA, fatigue with serial biopsies, or anxiety about forgoing treatment [Penson (2012), Dall'Era (2015)].

Active surveillance is the preferred approach for managing newly diagnosed, low-risk prostate cancer [Tosoian et al. (2016)]. However, there have been no randomized trials comparing prostate cancer mortality or other long-term outcomes under different active surveillance protocols. At present, information about active surveillance outcomes is based on prospective cohorts with limited followup. Among cohorts with the longest follow-up, reported risks of disease progression have been highly variable. However, it is unclear whether differences in the reported risks are due to underlying differences in participant selection or to differences in active surveillance implementation, including surveillance schedules, definitions of progression, and rates of dropout to treatment without progression. Clarifying whether risks of progression observed across cohorts persist after accounting for differences in implementation will provide valuable information about the representativeness of individual cohorts and about uncertainty of expected outcomes for newly diagnosed prostate cancer patients who are considering active surveillance. 
Most active surveillance cohorts define prostate cancer progression as an increase in tumor grade or volume on biopsy. Grade refers to the degree of cellular differentiation in the tumor, that is, the degree to which cancer cells resemble ordinary prostate cells. Prostate cancer grade is quantified by Gleason score, which reflects the degree of differentiation of the majority of tissue and the rest of the tissue in the tumor [Humphrey (2004)]. Gleason score is an established predictor of progression risk in treated and untreated prostate cancer patients [Popiolek et al. (2013)]. Low-grade cancer refers to Gleason score $\leq 6$. In this article, we define progression on active surveillance as an increase in Gleason score because this is a common component of the definition of progression in all active surveillance cohorts, whereas an increase in tumor volume is not consistently defined. Treatment for reasons other than tumor upgrading is considered to be a competing risk in that it prevents observing tumor upgrading in the absence of treatment.

We describe a method that accommodates differing biopsy frequencies across active surveillance cohorts and produces comparable, continuous-time projections of the risk of tumor upgrading in each cohort. In practice, the risks of upgrading and treatment are likely to be correlated: factors such as a rising PSA, which may induce a decision to initiate treatment, may also be related to the risk of progression [Ross et al. (2010)]. Our method accommodates this by modeling both the time to progression and the time to treatment as dependent on baseline PSA level and PSA velocity. In addition, the method accommodates as a fixed input the misclassification that occurs when biopsy grade is not an accurate reflection of the true, underlying grade (i.e., the pathological grade that would be assessed following surgery). Since biopsies only sample a limited portion of the prostate, they may be subject to misrepresentation of the true tumor biology. It has been estimated that in active surveillance cohorts, high-grade cancers are misclassified as low grade 10-50\% of the time [Palisaar et al. (2012), Inoue et al. (2014), Pinsky, Parnes and Ford (2008)]. Misclassification of a low-grade tumor as a high-grade tumor may occur up to $15 \%$ of the time [Inoue et al. (2014)]. We present results with and without misclassification of specified magnitudes.

Our approach considers serial biopsies to reflect discrete views of a continuoustime stochastic process with a discrete state space corresponding to low-grade (Gleason score $\leq 6$ ) and high-grade (Gleason score $\geq 7$ ) cancer while capturing early treatment as a competing risk state. The underlying disease progression is modeled as occurring according to a latent continuous-time Markov chain (CTMC), and we assume that the biopsies consist of discrete, possibly misclassified observations of the underlying process [Titman and Sharples (2010), Lange and Minin (2013)]. Latent CTMCs offer much more flexibility than standard CTMCs, which, due to their tractability, are frequently used to characterize discretely observed, multistate processes describing disease progression [Mandel (2010), Jackson et al. (2003)]. Further, the likelihood of latent CTMC models is analogous to a hidden Markov model likelihood, and therefore this framework naturally is able to incorporate misclassified disease outcomes. We link risk of progression and competing treatment via baseline PSA and PSA velocity, enabling us 
to estimate the risk of upgrading in the absence of treatment. We use our modeling framework to analyze individual-level data from four of the largest and most prominent North American active surveillance studies.

\section{Methods.}

2.1. Overview. In this section we describe the model framework that allows us to compare the four active surveillance cohorts in terms of their continuous-time underlying risks of progression. First we describe each of the active surveillance datasets. Next we detail the latent CTMC model used to describe the underlying upgrades: the competing risks structure for disease progression, the dependence of the event times on the evolving PSA, the mechanism for incorporating misclassification error in the biopsy observations, and the likelihood function. Finally, we describe a hypothesis test for comparing the risk of upgrading across cohorts.

2.2. Data sources. De-identified, individual-level datasets were obtained from four active surveillance cohorts following institutional review board approval.

1. The Johns Hopkins University (JHU) dataset [Tosoian et al. (2015)] consists of 913 men enrolled during 1994-2014. This study enrolled men with very low risk prostate cancer (clinical stage T1c, PSA density $\leq 0.15 \mathrm{ng} / \mathrm{ml}$, Gleason score $\leq 6, \leq 2$ positive biopsy cores, and $\leq 50 \%$ involvement of any biopsy core with cancer), as well as older men with low risk disease (clinical stage $\leq \mathrm{T} 2 \mathrm{a}$, PSA $<10 \mathrm{ng} / \mathrm{ml}$, and Gleason score $\leq 6$ ). Men were tracked with PSA tests every six months and had annual biopsies. Treatment intervention was recommended for disease reclassification, defined as any adverse grade or volume change detected on biopsy.

2. The Canary Prostate Active Surveillance Study (PASS) dataset [Newcomb et al. (2016)] consists of 1067 men enrolled during 2008-2013. Enrollment criteria included prostate cancer with clinical stage $\leq \mathrm{T} 2$ disease with no previous treatment and either a 10-core biopsy within one year before enrollment or $\geq 2$ biopsies with $\geq 1$ in the year before enrollment. Participants were followed with PSA measurements every four months and had repeat biopsies 6-12, 24, 48, and 72 months after enrollment. Treatment intervention was recommended if there was either an increase in biopsy Gleason score or volume detected on biopsy (from $<33 \%$ to $\geq 33 \%$ of cores positive for cancer).

3. The University of California San Francisco (UCSF) dataset [Welty et al. (2015)] consists of 1319 men enrolled during 1990-2015. Although eligibility criteria have evolved over time, the current criteria are PSA $\leq 10 \mathrm{ng} / \mathrm{ml}$, clinical stage $\leq \mathrm{T} 2$, biopsy Gleason score $\leq 6, \leq 33 \%$ positive cores, and $\leq 50 \%$ tumor in any single core. However, carefully selected cases who do not satisfy these criteria may be enrolled. Surveillance biopsies are recommended within the first year and every 12-24 months thereafter, sampling at least 12 biopsy cores. The primary trigger for treatment was biopsy grade reclassification. 
4. The University of Toronto (UT) dataset [Klotz et al. (2015)] consists of 1104 men enrolled during 1995-2015. During 1995-1999, the study was offered to all low-risk men (Gleason score $\leq 6$ and PSA $\leq 10 \mathrm{ng} / \mathrm{ml}$ ) and to men $>70$ years of age with PSA $<15 \mathrm{ng} / \mathrm{ml}$ or Gleason score $\leq 3+4$. During 2000-2015, the study also included men with favorable intermediate-risk disease (PSA $\leq$ $20 \mathrm{ng} / \mathrm{ml}$ and/or Gleason score $\leq 3+4$ ) with significant comorbidities and a life expectancy $<10$ years. PSA tests were performed every three months for two years and then every six months in stable men. A confirmatory biopsy was performed within 12 months of the initial biopsy and then every three-four years until age 80. Treatment intervention was recommended if there was an upgrade in histology on repeat biopsy or clinical progression between biopsies. Until 2009, intervention was also recommended on the basis of having a PSA doubling time $<$ three years.

Records from each dataset included diagnosis years, patient ages, clinical and pathologic information at diagnosis, dates and results of all surveillance tests, including PSA values and biopsy results, dates and types of curative treatment, and vital status. Patients diagnosed before 1995, older than 80 years at enrollment, or with Gleason score $\geq 7$ at diagnosis were excluded from the analysis in order to create a more homogeneous population across the four cohorts.

To enforce consistency, we defined disease progression strictly in terms of grade progression, that is, the first point at which a Gleason score $\geq 7$ was reached. For our analyses, we differentiate between the time of underlying upgrade (UGC) and observed upgrade, where the former refers to the unobserved time when the cancer progresses from Gleason score $\leq 6$ to Gleason score $\geq 7$ and the latter to the observed time when grade is reclassified on biopsy. We refer to the Gleason score $\leq 6$ as "low grade" and Gleason score $\geq 7$ as "high grade." We also define the event of treatment without grade reclassification as the initiation of treatment in the absence of an observed biopsy upgrade. Volume reclassification or patient choice may also trigger treatment without observed upgrading. We refer to treatment without observed upgrading as "competing treatment" since its initiation precludes observing upgrading on active surveillance. One can view this competing treatment as a form of dependent censoring that may be correlated with the time of underlying upgrade.

\subsection{A model for continuous-time prostate cancer progression.}

2.3.1. Model overview. Patients enter the active surveillance cohort just following diagnosis of low-grade disease. Their data consists of a follow-up sequence of biopsy times and Gleason score results, a sequence of PSA test times and results, and the time of competing treatment, if any (Figure 1A). The end of follow-up occurs at the minimum of the time that the first biopsy detects high-grade cancer, the time that competing treatment is initiated, or the time of the last PSA test in the absence of either of these events. Let $t_{k 0}=0$ be the time of the original cancer 
A.

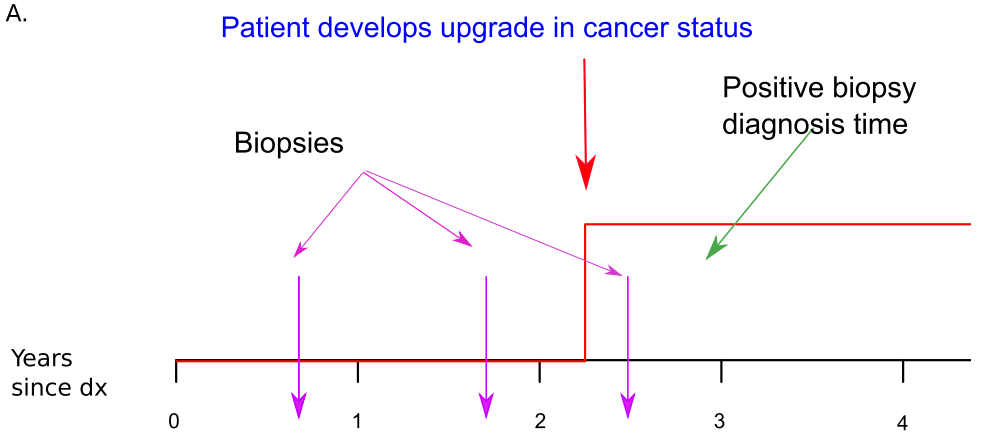

B.

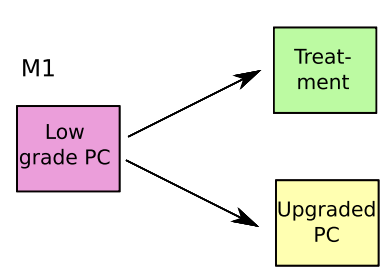

C.

D.

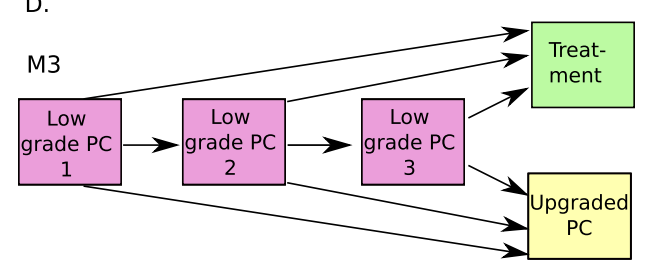

FIG. 1. A. Sample trajectory for a patient who upgrades during the observation period. The biopsies detect the underlying process at discrete times, and the time of diagnosis occurs after the time of underlying upgrade. B. Competing risks structure of the model describing cancer grade progression or competing treatment modeled as a standard CTMC, denoted M1. C. Latent continuous time Markov chain (CTMC) structure with two low-grade cancer states that generates non-constant hazard rates for transitions, denoted M2. D. Latent CTMC structure with three low-grade cancer states, denoted M3.

diagnosis for patient $k ; t_{k 1}, \ldots, t_{k n}$ be the biopsy times; and $o_{k 1}, \ldots, o_{k n}$ be the Gleason score results. Let $t_{k 1}^{\mathrm{psa}}, \ldots, t_{k m}^{\mathrm{psa}}$ be the PSA test times and $y_{k 1}, \ldots, y_{k m}$ be the results (PSA values on the log-scale). Let $h_{k}$ be an indicator of whether the final observation time corresponds to a biopsy $\left(h_{k}=1\right.$ if yes and $h_{k}=0$ otherwise). If $h_{k}=0$, let $z_{k}$ be the end of follow-up, which may be either the time of competing treatment (in which case $c_{k}=1$ ) or the final PSA test (in which case $\left.c_{k}=0\right)$.

These data are used to inform a model of continuous-time prostate cancer grade progression involving three components. 
1. A random effects model captures log-PSA growth for subject $k$ at time $j \in$ $\{1,2, \ldots, m\}$ as follows:

$$
Y_{k j}=\gamma_{0 k}+\gamma_{1 k} t_{k j}^{\mathrm{psa}}+\varepsilon_{k j}
$$

where $\left(\gamma_{0 k}, \gamma_{1 k}\right)$ are normally distributed subject-level random effects and $\varepsilon_{k j}$ is a zero-mean, normally distributed, within-subject error term.

2. A competing risks model for underlying upgrading and competing treatment based on a latent continuous-time Markov chain (CTMC), as described in Section 2.3.2. Transition rates in the model depend on baseline age and PSA intercepts and slopes estimated in advance of fitting the competing risks model. Times of underlying upgrading and competing treatment are assumed to be conditionally independent given baseline age and PSA intercept and slope.

3. A misclassification model governing imperfect sensitivity of biopsies to detect high-grade disease, as described in Section 2.3.6.

2.3.2. A competing risks model for underlying upgrade. In the competing risks model structure, individuals proceed from low-grade cancer to either highgrade cancer or competing treatment. We can characterize the trajectory through underlying disease states as a multistate process, $W(t)$, with state space $R=$ $\{1,2,3\}$, where state 1 is the low-grade state, state 2 is the high-grade state, and state 3 is the competing treatment state. At each biopsy, $W(t)$ corresponds to the true underlying state, so that $W\left(t_{k 0}\right), \ldots, W\left(t_{k n}\right)$ reflect discrete snapshots at times $t_{k 0}, \ldots, t_{k n}$.

As a first pass, we might specify $W(t)$ as the simplest of multistate models, a time-homogeneous CTMC with state space $R$. The Markov property of this model means that transitions between states at any given time depend only on the state occupied at that time and not on the history of the process before that time. Time homogeneity means that the probability of transitioning from state $i$ to state $j$ between times $s$ and $s+t$ is the same as the probability of transitioning between these states at times 0 and $t$. We specify a CTMC $W(t)$ by a transition intensity matrix $\boldsymbol{\Lambda}=\left\{\lambda_{i j}\right\}$, where $\lambda_{i j}$ refers to the instantaneous transition rate between state $i$ and $j$, and an initial distribution $\pi$ that specifies the probability of occupying each state at time 0 .

Suppose that $\mathbf{P}(t)=\left\{\mathrm{P}_{i j}(t)\right\}$ is the matrix representing transition probabilities between states $i$ and $j$ in the interval $[s, s+t]$. The transition probability matrices between states are characterized by a matrix exponential of the intensity matrix $\boldsymbol{\Lambda}$,

$$
\mathbf{P}(t)=\exp (\boldsymbol{\Lambda} t) .
$$

In general, the density of the time transition to state $k$ at time $s+t$, given that the process is in state $i$ at time $s$, is:

$$
f_{i k}(t)=\sum_{j} \mathrm{P}_{i j}(t) \lambda_{j k} .
$$


Transition probabilities can be computed with standard methods [Moler and Loan (2003)].

While standard CTMCs have appealing analytic tractability, they assume that sojourn times (i.e., durations spent in a state before transitioning) are exponentially distributed and that rates of transitioning between states are constant with respect to sojourn duration. In our setting, this assumption is unrealistic. Therefore, to enable more flexible sojourn time distributions, we assume that the disease process $W(t)$ is based on an underlying latent CTMC $X(t)$ with state space $S$, where multiple latent states in $S$ may map to each observable disease state in $R$. We do not assign biological meaning to the latent states in the model but simply use them as a tool for more flexible sojourn time distributions.

In particular, the state space is $S=\left\{1_{1}, \ldots, 1_{s_{1}}, 2,3\right\}$, where $s_{1}$ is the number of latent low-grade states. The mapping of $S$ into $R$ is:

$$
\begin{aligned}
& W(t)=1 \quad \Leftrightarrow \quad X(t) \in\left\{1_{1}, \ldots, 1_{s_{1}}\right\}, \\
& W(t)=2 \quad \Leftrightarrow \quad X(t)=2, \\
& W(t)=3 \quad \Leftrightarrow \quad X(t)=3 .
\end{aligned}
$$

The latent CTMC sojourn time distribution in the low-grade cancer state $[W(t)=1]$ can be thought of a as mixture of all of the possible paths out of that state, which allows for more flexibility than exponential sojourn times.

2.3.3. Model selection. Figure 1A represents the structure of a standard continuous time Markov chain $\left(s_{1}=1\right)$, and Figures 1C and 1D depict the more flexible latent structures that we consider for the prostate cancer progression model. In general the coarseness of data provides practical limits on the number of latent states it is advisable to fit, and we recommend people start with smaller models and build up as the data permits, stopping when the model estimation is numerically unstable. For this setting, we considered standard CTMCs $\left(s_{1}=1\right)$ and latent CTMCs with $2\left(s_{1}=2\right)$ and 3 low-grade states $\left(s_{1}=3\right)$, which we refer to as $M 1$, $M 2$, and $M 3$, respectively. We use the Bayesian information criterion (BIC) as a means of choosing the number of latent states given its good performance in choosing the number of components in latent models [Steele and Raftery (2010)].

2.3.4. Incorporation of patient covariates. We incorporate individual age and PSA intercept and slope $\left(\gamma_{0 k}, \gamma_{1 k}\right)$ as covariates into $\boldsymbol{\Lambda}=\left\{\lambda_{i j}\right\}$, the transition matrix for the latent CTMC $X(t)$ in the competing risk model. Including these covariates in the model induces a dependence between times of underlying upgrading and competing treatment. To do so, we relate log-rates to a linear predictor, $\log \left(\lambda_{i j}^{(k)}\right)=\zeta_{i j}^{T} \mathbf{v}^{(k)}$, where $\mathbf{v}^{(k)}$ is the vector of covariates for patient $k$. We incorporate covariates in this way for each possible transition out of the low-grade state to the high-grade state and to the competing treatment state, that is, for each $\lambda_{i j}$ where $i \in\left\{1_{1}, \ldots, 1_{s_{1}}\right\}$ and $j \in\{2,3\}$. 
For parameter identifiability, we assume that the covariate effect for transitions from low-grade to high-grade states is the same (i.e., for each $\lambda_{i, 2}$ where $\left.i \in\left\{1_{1}, \ldots, 1_{s_{1}}\right\}\right)$ and similarly for the transition from low-grade to competing treatment states (i.e., for each $\lambda_{i, 3}$ where $\left.i \in\left\{1_{1}, \ldots, 1_{s_{1}}\right\}\right)$. Note that unless $W(t)$ is a standard CTMC, this specification does not necessarily imply proportional hazards [e.g., Marshall and Jones (1995)]. Therefore, rather than interpreting the covariate parameter estimates directly, it is more revealing to visualize covariate effects on the risk of underlying upgrading.

\subsubsection{Projecting the cumulative distribution of underlying upgrade times in ab-} sence of competing treatment. Assuming that underlying upgrade and competing treatment are conditionally independent given patient covariates, we can project the distribution of time to underlying upgrade in absence of treatment by eliminating competing treatment in the transition intensity matrix. This involves creating a new transition matrix $\tilde{\Lambda}=\left\{\tilde{\lambda}_{i j}\right\}$, which is identical to $\boldsymbol{\Lambda}$ except that transition rates to the competing treatment state $\lambda_{i 3}$, where $i \in\left\{1_{1}, \ldots, 1_{s 1}\right\}$, are set to zero. The distribution function for underlying upgrade in the absence of competing treatment, starting from the first low-grade state, is thus provided by the row of the transition probability matrix

$$
\tilde{\mathbf{P}}(t)=\exp (\tilde{\mathbf{\Lambda}} t)
$$

corresponding to the transition between state $1_{1}$ and state 2 .

2.3.6. Biopsy misclassification. An emission matrix $\boldsymbol{E}=\{e(i, j)\}$ characterizes the relationship between the observed biopsy Gleason score and the underlying state $X(t)$ and has entries $e(i, j)=\mathrm{P}\left(O_{t}=j \mid X(t)=i\right)$. If there is no biopsy misclassification, the emission matrix simply maps the state space of $W(t)$ to the state space of $X(t)$. Otherwise, the emission matrix describes the probability of the observed biopsy outcomes given the underlying states in $W(t)$, assuming that the observed biopsies are conditionally independent given the values of the underlying state at each observation time. The emission matrix is given by

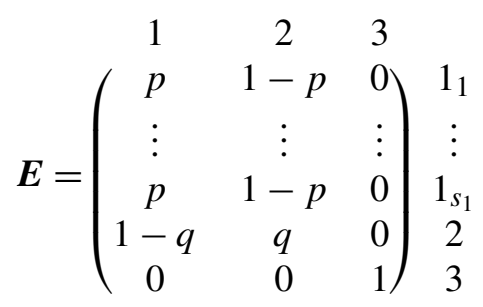

where $q$ is biopsy sensitivity (i.e., the probability of observing upgrading if underlying upgrading has occurred) and $p$ is the biopsy specificity (i.e., the probability of not observing upgrading if no upgrading has occurred). In practice it is challenging to estimate biopsy misclassification jointly with the transition rate parameters 
in a competing risks multistate process without some observations corresponding to a gold standard [Inoue et al. (2014)]. To investigate the impact of biopsy misclassification, we perform analyses assuming no biopsy misclassification error and compare results when biopsy sensitivity is $60 \%, 75 \%$, and $90 \%$, and biopsy specificity is $95 \%, 90 \%$, and $85 \%$, which encompass the range presented in the literature [Inoue et al. (2014)].

2.3.7. Initial state distribution. The initial state distribution $\pi$ with entries $\pi_{j}=P\left(X\left(t_{0}\right)=j\right)$ represents the probabilities of underlying states at diagnosis. In our dataset, the first biopsy occurs $\geq 6$ months after diagnosis, so this initial state distribution is an extrapolation. If we assume there is no biopsy misclassification, then biopsy Gleason score at diagnosis correctly identifies all patients with low-grade disease. Per the assumptions of the Coxian sojourn time distribution [Cumani (1982)], individuals with low grade disease initially occupy the first latent state: $\pi_{1_{1}}=1$. If we allow biopsy misclassification, we assume that individuals start either in state $1_{1}$ or in state 2 , and all other initial probabilities are zero. In this case, the initial distribution is estimated from a logistic regression model that depends on patient age and PSA intercept $\left(\gamma_{0 k}\right)$ :

$$
\log \frac{\pi_{2}}{\pi_{1_{1}}}=\beta_{1}+\beta_{2} \gamma_{0 k}+\beta_{3} \text { age }_{k}
$$

2.3.8. Likelihood of the observed data. Suppressing the individual subscripts, a patient's observed data vector is $\boldsymbol{o}=\left(o_{1}, \ldots, o_{n}, h, c, z\right)$, reflecting the observed biopsy results, an indicator for whether his final observation was a biopsy, an indicator for whether he initiated competing treatment, and the end of follow-up (see Section 2.3.1). His underlying disease states corresponding to his status at the entry into active surveillance at diagnosis and each of the follow-up biopsy times is $\left(x_{0}, \ldots, x_{n}\right)$. If there were no latent states or misclassification error, the likelihood would be a product of conditional probabilities of the observed data at each time, given the previous observed data. In a general case, where we assume a latent CTMC model, or a model with misclassification error, we need to marginalize (sum) the product of conditional probabilities across the hidden states to obtain the likelihood of the observed states. In this sense it resembles a hidden Markov model which marginalizes the joint probability of the underlying disease states $\left(x_{0}, \ldots, x_{n}\right)$ and the observed data at the corresponding times across $\left(x_{0}, \ldots, x_{n}\right)$ :

$$
\begin{aligned}
\mathrm{P}(\boldsymbol{o})= & \sum_{x_{0}} \sum_{x_{1}} \cdots \sum_{x_{n}} \pi_{i} \prod_{i=0}^{n-1} \mathrm{P}_{x_{i} x_{i+1}}\left(t_{i+1}-t_{i}\right) \prod_{i=1}^{n} e\left(x_{i}, o_{i}\right) \\
& \times\left(\left[f_{x_{n} 3}\left(z-t_{n}\right)\right]^{c}\left[\sum_{j \neq 3} \mathrm{P}_{x_{n} j}\left(z-t_{n}\right)\right]^{1-c}\right)^{1-h} .
\end{aligned}
$$

Here $f_{x_{n} 3}\left(z-t_{n}\right)$ is the density function for competing treatment at time $z$ given underlying grade at the final biopsy, and $\sum_{j \neq 3} \mathrm{P}_{x_{n} j}\left(z-t_{n}\right)$ is the probability of not initiating competing treatment at time $z$. 
2.3.9. Parameter estimation and software implementation. We obtain the maximum likelihood estimates for model parameters $\boldsymbol{\theta}=(\boldsymbol{\pi}, \boldsymbol{\Lambda})$ using an expectation-maximization (EM) algorithm that uses quantities from the BaumWelch algorithm for obtaining estimates for a discrete-time hidden Markov model [Baum et al. (1970)]. The algorithm is described in Lange and Minin (2013) and is recapped in Supplement A [Lange (2018)]. The algorithm is implemented using our R package, cthmm, available at http://r-forge.r-project.org/projects/ multistate/. To obtain variance estimates for the model parameters, we use numerical estimation of the observed Fisher information matrix using the R package "NumDeriv" [Gilbert and Varadhan (2012)].

2.4. Comparisons across cohorts. Our primary goal is to compare rates of underlying upgrading across active surveillance cohorts. To do so, we first combine data from the cohorts and parameterize the rates of underlying upgrade and competing treatment using a dummy variable for cohort as a covariate. Given estimated cohort effects, we use Wald tests to evaluate the statistical significance of differences in rates of underlying upgrading across cohorts.

\section{Results.}

3.1. Cohort summary and empirical outcomes. After exclusions, the sample consists of 699, 613, 764, and 421 patients from JHU, PASS, UCSF, and UT, respectively. Distributions of baseline characteristics, surveillance biopsy and PSA test frequencies, and outcomes across the cohorts are shown in Supplement B, Table 1 [Lange (2018)]. JHU had the most frequent biopsies (median of 1 per 0.9 years) and UT the least frequent biopsies (median of 1 per 3.8 years); both PASS and UCSF had a median of 1 per 1.8 years.

Figure 2 shows the empirical cumulative incidence curves of observed upgrading and competing treatments across cohorts, each derived using the other event as a competing risk. Figure 2A shows that the PASS and UCSF cohorts had the highest empirical cumulative incidence of observed upgrading, while JHU had the lowest. The 10-year empirical cumulative incidence in PASS and UCSF was 59\%, in JHU was $27 \%$, and in UT was $45 \%$. Figure $2 \mathrm{~B}$ shows that JHU had the highest risk of competing treatment (10-year empirical cumulative incidence is $27 \%$ ), possibly due to the relatively high incidence of volume-only progression in this cohort, which accounts for about half the cases reclassified, whereas the other cohorts had similar, lower risks of competing treatment (10-year empirical cumulative incidences ranged from 9-12\%).

Based on the PSA growth models, median PSA velocity was similar across the cohorts, with $1 \%$ annual increase (IQR -3\%, 6\%) for JHU, 4\% (IQR - 3\%, 11\%) for PASS, 4\% (IQR -1\%, 10\%) for UCSF, and 5\% (IQR - 2\%, 11\%) for UT.

\subsection{Models of continuous-time prostate cancer progression.}

3.2.1. No biopsy misclassification. Table 1 presents the results of fitting standard and latent CTMC models to each cohort. When no biopsy misclassification 

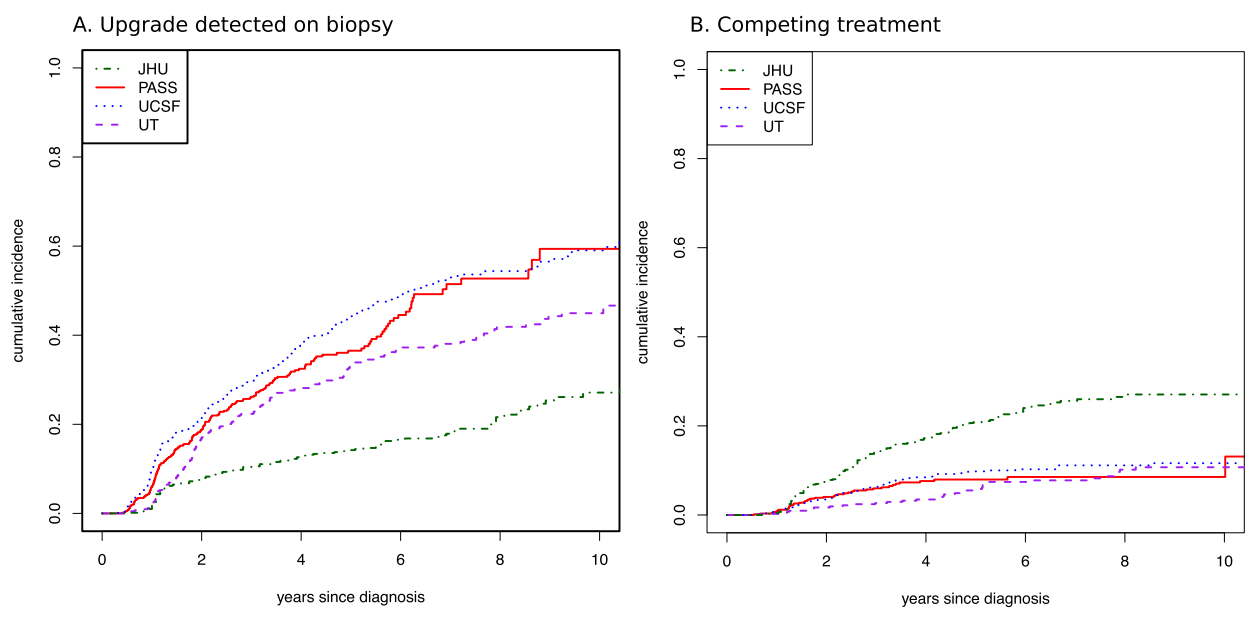

FIG. 2. A. Empirical cumulative incidence of observed upgrading in the presence of competing treatment. B. Empirical cumulative incidence of competing treatment in the presence of observed upgrading.

was assumed, PASS, UCSF, and UT all achieved the best fit (according to the BIC) under model M2 $\left(s_{1}=2\right)$; JHU achieved the best fit under model M3 $\left(s_{1}=3\right)$. Parameter estimates are presented in Supplement C, Table 1 [Lange (2018)]. Using best fitting models for each cohort, Figure 3 presents the predicted cumulative probability of continuous-time underlying upgrading in the absence of competing treatment in each cohort based on averaging individual-level results. Naive Kaplan

TABLE 1

Model selection assuming no biopsy misclassification. Abbreviations: BIC = Bayesian Information Criterion; M1, M2, M3 refer to standard CTMC and models with 2 and 3 latent states, respectively

\begin{tabular}{lccccc}
\hline Cohort & No. patients & Latent CTMC model & No. params & Log likelihood & BIC \\
\hline JHU & \multirow{2}{*}{699} & M1 & 8 & -1086.4 & 2195.6 \\
& & M2 & 11 & -1069.6 & 2170.5 \\
\multirow{3}{*}{ PASS } & \multirow{2}{*}{613} & M3 & 14 & -1055.8 & 2151.4 \\
& & M1 & 8 & -688.1 & 1398.5 \\
& \multirow{3}{*}{764} & M2 & 11 & -677.1 & 1384.9 \\
UCSF & M3 & 14 & -674.8 & 1388.6 \\
& & M1 & 8 & -1149.4 & 2321.8 \\
& \multirow{3}{*}{421} & M2 & 11 & -1122.0 & 2275.7 \\
UT & M3 & 14 & -1122.0 & 2284.4 \\
& & M1 & 8 & -495.3 & 1011.6 \\
& & M2 & 11 & -480.4 & 989.7 \\
& & M3 & 14 & -479.2 & 995.1 \\
\hline
\end{tabular}




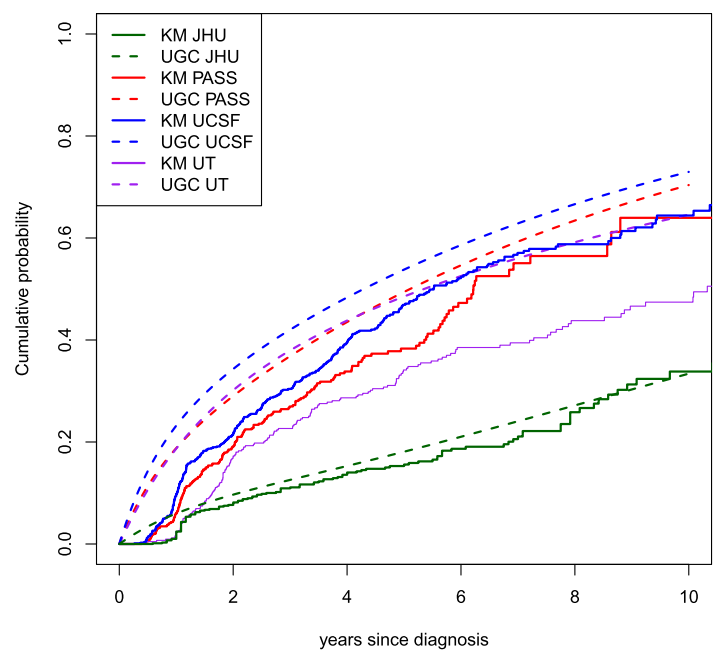

FIG. 3. Comparison of observed upgrading (solid Kaplan-Meier curves) and underlying upgrading (dashed continuous-time model predictions) in the absence of competing treatments assuming no biopsy misclassification.

Meier (KM) based estimates of cumulative distribution functions are included for comparison purposes. In general, the KM curves are shifted right relative to the continuous-time predictions, consistent with the expectation that underlying upgrading precedes observed upgrading. The magnitude of this shift depends on the biopsy frequency, with more frequent biopsies yielding a smaller shift.

Comparing underlying upgrading in the absence of competing treatment across cohorts indicates that JHU has a markedly lower rate than the other three cohorts. We estimate that $33 \%$ of patients in JHU had an underlying upgrade within 10 years, but this probability is $65-73 \%$ in the other cohorts. This difference is highly significant $(p<0.001$ from Wald test for any differences across cohorts based on the model fit to combined cohort data), as are pairwise differences between JHU and the other cohorts (all $p<0.001$ ). Pairwise differences between PASS, UCSF, and UT are also significant, even after Bonferroni correction for multiple comparisons, except for the difference between PASS and UT $(p=0.31)$. These observations differ considerably from the impression given by the empirical curves. In contrast, a naive cohort comparison based on a Cox regression (which ignores both the differences in surveillance frequency and dependent competing risks) suggests that UT has a significantly lower hazard of upgrading relative to PASS (hazard ratio $=0.61, p<0.001)$. All other pairwise comparisons are also significant, except for PASS and UCSF (hazard ratio $=1.17, p=0.08$ ).

3.2.2. Allowing for biopsy misclassification. We first examined how biopsy sensitivity affects estimated probabilities of underlying upgrade, assuming biopsies ere $60 \%, 75 \%$, and $90 \%$ sensitive and $100 \%$ specific. The BIC-selected latent 
structures were the same as for the models fit assuming no misclassification error. With imperfect biopsy sensitivity, some patients may have had high-grade disease that was not detected at the time of diagnosis. We fit these models (1) assuming all patients had low-grade disease at entry and (2) allowing a non-zero probability of high-grade disease at entry. In the standard CTMC model, allowing a non-zero probability of high-grade disease at entry substantially improved the fit. However, in the latent CTMC models, allowing a non-zero probability of high-grade disease at entry did not improve fit. These results are shown in Supplement D, Tables 1-4 [Lange (2018)], where the maximum likelihood values are virtually identical for the latent CTMC models with zero and non-zero probabilities of high-grade disease at entry. Moreover the MLEs for this probability are zero. As discussed in Bladt and Sorensen (2005), the CTMCs under discrete observations may have MLEs that fall on the boundary of the parameter space or may not exist if the observations are not sufficiently frequent. Thus it is likely that the latent CTMCs did not have sufficient data to estimate the probability of high-grade disease at enrollment. To understand this issue further, we plotted the estimated cumulative probability of underlying upgrading assuming a $60 \%$ biopsy sensitivity under a model with one and under a model with two or three low-grade states (Supplement E, Figure 1 [Lange (2018)]). The figure shows that there is little difference between the two model projections after the median time of first active surveillance biopsy in each cohort, suggesting that the latent CTMC model can explain the observed data equally well with or without a non-zero probability of high-grade disease at enrollment.

Figure 4 presents underlying upgrading incidence for selected biopsy sensitivity rates (and $100 \%$ specificity), averaged across the individuals in each cohort. In general, when biopsies are less sensitive, the predicted underlying upgrade occurs sooner. This is because lower biopsy sensitivity implies a lower detection of the underlying condition so that the true frequency of the underlying condition must be higher than that observed. The impact of different biopsy sensitivities on the estimated probability of high-grade disease was more pronounced at earlier biopsies. The impact of biopsy sensitivity also varied across cohorts, with JHU exhibiting the smallest impact and UT the largest, consistent with the ordering of biopsy frequency across cohorts. Even assuming a relatively low biopsy sensitivity, in JHU less than $10 \%$ were estimated to have true high grade disease one year after they entered the cohort, in contrast to the other cohorts where $20-45 \%$ were estimated to have true high grade disease at the same time.

Figure 5 presents underlying upgrading incidence for selected biopsy specificity rates (and 100\% sensitivity), averaged across the individuals in each cohort. In general, lower specificity implies a higher detection of underlying upgrading (more false positives) so that the true frequency of underlying upgrading must be lower than that observed. In JHU, under $90 \%$ and $85 \%$ biopsy specificity, the incidence of true underlying upgrade is estimated to be $0 \%$ across the follow-up period. Under 
A. JHU

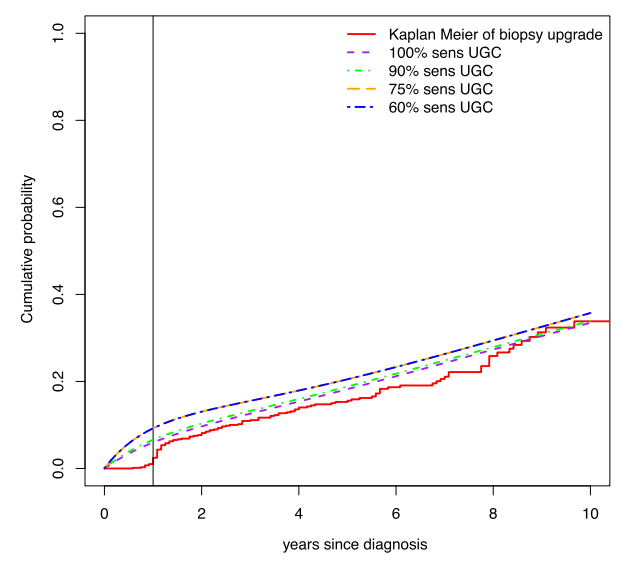

C. UCSF

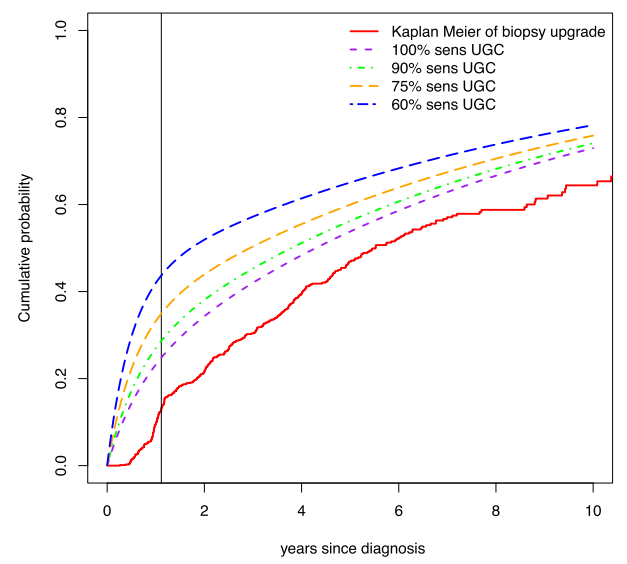

B. PASS
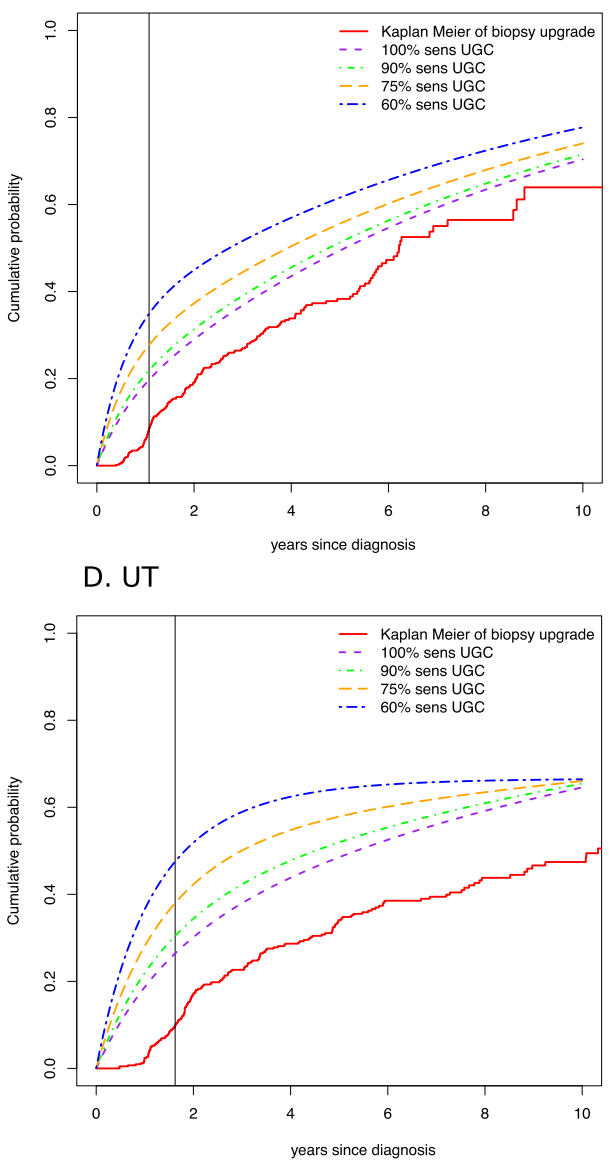

FIG. 4. Underlying upgrading under varying fixed levels of biopsy sensitivity, assuming $100 \%$ specificity, averaged across individuals in the cohort. Vertical lines show the median time of the first active surveillance biopsy in each cohort.

a yearly schedule a $10 \%$ or higher false positive rate on each biopsy is substantial enough to account for all of the observed biopsy upgrades in this cohort.

Finally, we estimated the probability of underlying upgrade in each cohort assuming 60\% biopsy sensitivity and $85 \%$ specificity [Inoue et al. (2014)]. Results of these analyses are shown in Figure 6 along with naive KM based estimates of biopsy upgrades. Notably, JHU is still substantially lower than the other cohorts, which are relatively concordant.

4. Discussion. The problem of comparing results from multiple studies in which observation schedules differ has been referred to as a "twenty first century Tower of Babel" since studies with different surveillance schema are very 
A. JHU

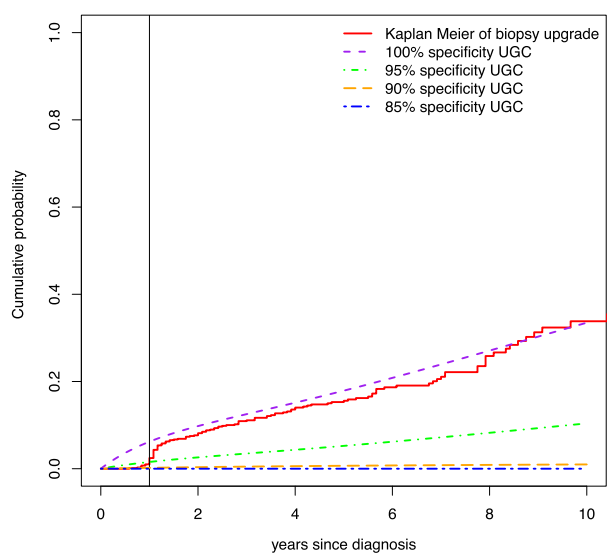

C. UCSF

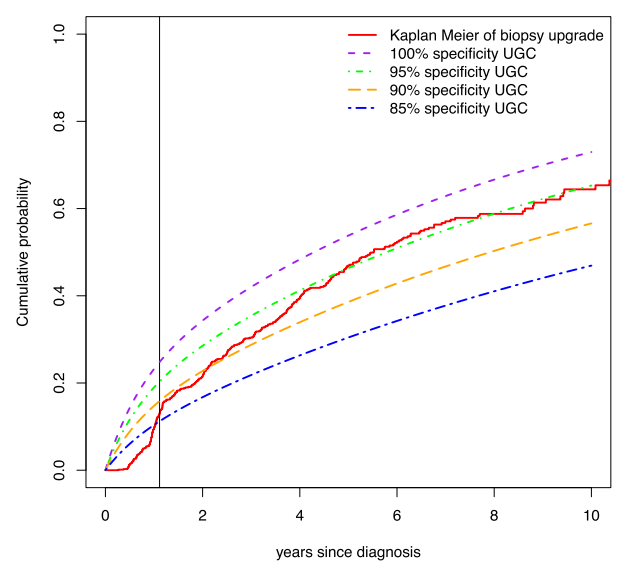

B. PASS

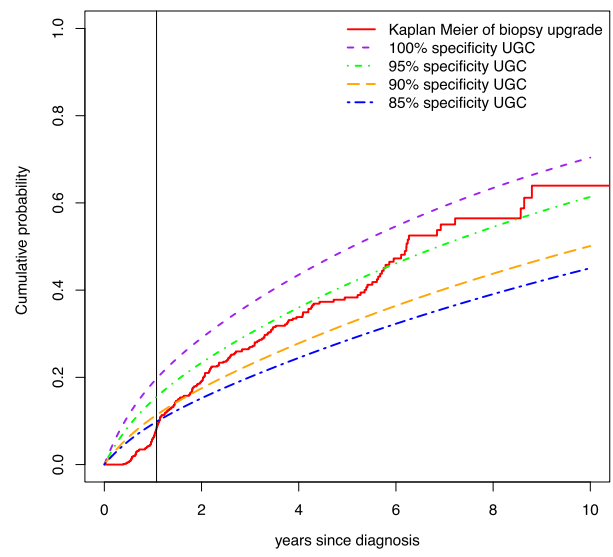

D. UT

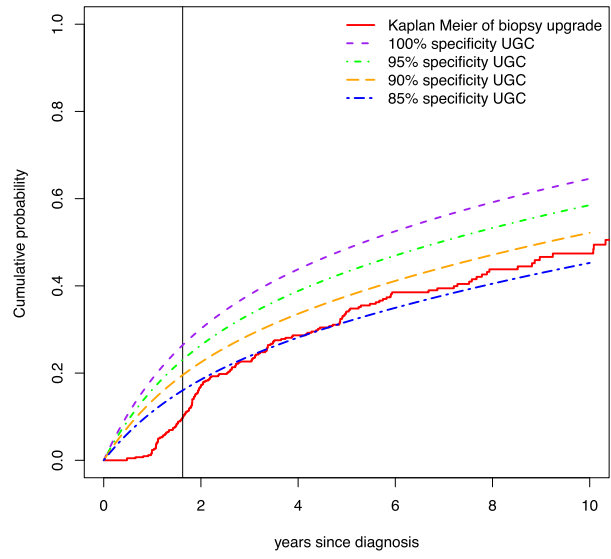

FIG. 5. Underlying upgrading under varying fixed levels of biopsy specificity, assuming $100 \%$ sensitivity, averaged across individuals in the cohort. Vertical lines show the median time of the first active surveillance biopsy in each cohort.

difficult to compare [Gignac et al. (2008)]. In the case of active surveillance for low-risk prostate cancer, a number of studies have reported disease progression risks for different cohorts with different definitions of progression and different biopsy schedules [Tosoian et al. (2016)]. To adequately compare the reported results, it is necessary to derive estimates under a consistent definition of progression and on a time scale that is the same for all studies. The latent CTMC multistate modeling approach presented here does exactly this, allowing us to characterize the risk of underlying upgrade, an outcome that means the same thing across studies. The method represents a novel alternative to a more standard meta-analysis of the empirical time to biopsy upgrade. The results are a proof-of-principle that the 


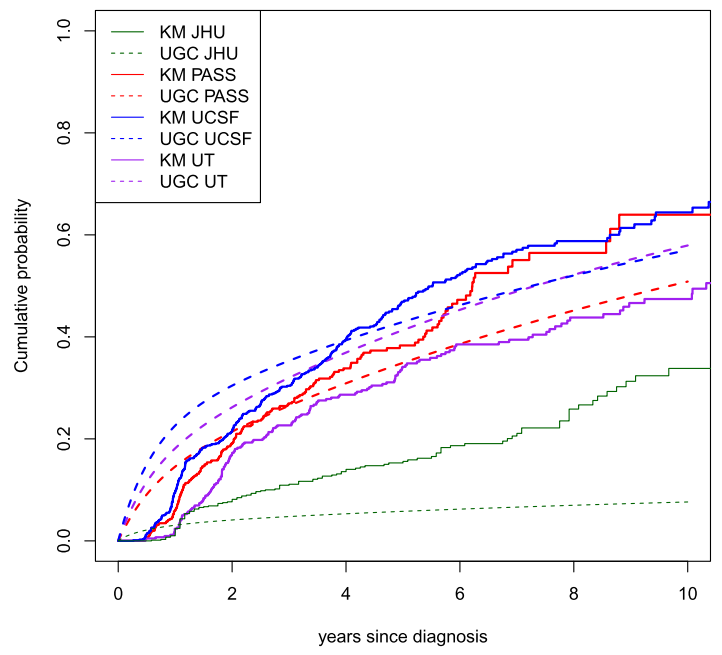

FIG. 6. Comparison of observed upgrading (solid Kaplan-Meier curves) and underlying upgrading (dashed continuous-time model predictions) in the absence of competing treatments assuming biopsies are $60 \%$ sensitive and $85 \%$ specific at detecting upgraded disease.

underlying, continuous-time patterns of biopsy upgrade across cohorts are not the same as the ones suggested by the empirical results.

Our results have implications for both clinical practice and policy development because they mandate caution when basing clinical predictions and/or recommendations on any single study. It appears that even when studies are made comparable in terms of outcomes and time scales, they do not always concur. The lower risk of underlying upgrade among JHU participants may be due to the fact that they were also selected on the basis of low PSA density (i.e., PSA level relative to prostate volume) and tended to have low volumes of cancer in their biopsy specimens. UCSF did not have a PSA density threshold for entry and included some cases with relatively high-volume disease in terms of the percent of biopsy cores with cancer. Thus, differences in inclusion criteria not captured in the available data could explain the persistent differences across studies.

A feature of the latent CTMC modeling approach is that it accommodates biopsy misclassification. We examine the impact of selected biopsy sensitivities and specificities on our results rather than simultaneously estimating these parameters since they are not identifiable without a gold standard result [Inoue et al. (2014)]. Our results demonstrate that the estimated rates of underlying upgrading can depend on biopsy accuracy. We found that assuming a low biopsy sensitivity was most compatible with the conclusion that a high fraction of patients have misclassified high-grade disease at the first active surveillance biopsy, but there is little change in underlying grade over time. In contrast, if we assume a high biopsy sensitivity, we estimate a low fraction of patients with high-grade disease at the 
first active surveillance biopsy and a faster rate of grade progression over time. Additionally, lower specificity (i.e., a higher false positive rate), leads to lower estimated rates of grade progression. Data sets that include surgical grade results will provide a gold standard and enable estimation of sensitivity and specificity in surveillance cohorts, which should provide better information on the true rates of grade progression over time.

By decoupling the underlying process of disease progression from the observed outcomes, our model accommodates both the biological process of underlying upgrading and the biopsy misclassification that contributes to the observed risk of upgrading. This property of our model contrasts with a recent study [Coley et al. (2016)] that effectively assumes non-changing underlying grade and imputes this grade on the basis of a Bayesian predictive model based on the subset of patients who went on to have surgery. Like Coley et al. (2016) our modeling approach also has the potential for use as a dynamic prediction tool; given accumulated observed data, one can calculate the underlying probability of having high-grade disease at a particular time and then predict state transitions beyond that time.

While our method provides novel insights, it is subject to some limitations. We assume that the events of treatment and underlying upgrade are correlated only via patient age and PSA intercept and slope. Failure to account for other factors associated with both events may lead to bias in estimates of the upgrading distribution in absence of competing treatment [Huang and Wolfe (2002)]. In particular, if we are ignoring factors that are positively correlated with upgrading and entering competing treatment, then our method would underestimate the upgrading risks, since men with higher treatment rates would enter competing treatment before their upgrading event was observed. Furthermore, rather than using joint modeling we incorporate PSA via a two-step approach: first estimating random effects and then using them as covariates in our disease progression model. This method may under-represent uncertainty and lead to some bias in projections of underlying upgrade, but has been used by others with latent CTMC models [Donnelly et al. (2017)]. Another implicit assumption is that individuals who undergo treatment without upgrading do not have an undetected grade progression before treatment. While this is not an issue for patients treated due to increases in tumor volume on their final biopsy, it could be relevant for cases treated between scheduled biopsies. Failure to account for progression in these patients could lead to an underestimate of the risk of underlying upgrade. A further limitation is that we were not able to estimate the misclassification probabilities jointly with the disease upgrading rates. Estimation of misclassification rates jointly with other parameters has been successful in other uses of the latent CTMC model under panel observation, albeit with substantially lower rates of misclassification than are likely in the prostate cancer biopsy setting [Titman and Sharples (2010)]. Despite this limitation, being able to project results under different misclassification probabilities clearly provides useful information and highlights the importance of this information in interpreting empirical results from active surveillance studies. 
Finally, we do not consider the possibility of informative observation times, which could be a feature of studies with a more relaxed biopsy protocol, such as UT. In a discretely observed multistate model, observation times are ignorable if they correspond to visits that are scheduled in advance, including those based on a patient's prior history of observed data [Grüger, Kay and Schumacher (1991)]. However, if visits are patient-initiated and depend on the underlying disease status, visit times are non-ignorable. Our prior simulation work has shown that when observations are more frequent if patients are in diseased states, ignoring informative visit times will lead to overestimates of the rates of transitioning between healthy and diseased states [Lange et al. (2015)]. In the active surveillance setting, it is unlikely that patients are symptomatic even if they do upgrade. Adherence or lack of adherence to protocol biopsies may be related to the prior history of biopsy and PSA results, but it is unlikely to additionally depend on the underlying grade. In future work, it may be worth investigating whether allowing for informative visit times changes our conclusions about times of upgrading, perhaps using the expansion of the model we previously developed [Lange et al. (2015)].

We note that others have developed methods that could be applied in the analysis of active surveillance data. Most relevantly, Mao, Lin and Zeng (2017) recently extended the nonparametric current status data methods [Hudgens, Satten and Longini (2001)] for an arbitrary sequence of examination times and time varying external covariates. In addition, Rouanet et al. (2016) provided a latent class model approach for longitudinal data and interval censored competing events. However, neither of these methods incorporates misclassification error as an input parameter.

More broadly, there are other statistical methods that can be used to model discretely observed continuous-time multistate processes with non-constant hazard functions for transitions between states. Semi-Markov multistate models represent one such approach, but options for these models are limited by the structure of the model and completeness of the observations. Data that are interval censored permit semi-Markov models that can be estimated via either parametric [Foucher et al. (2007)] or nonparametric [Frydman and Szarek (2009)] means. Panel data, for which both transition times and states are unknown in the inter-observation interval, present more difficulties since calculating transition probabilities requires integrating over all possible trajectories connecting states $i$ and $j$ on a given time interval. In particular, semi-Markov models for panel data for a general multistate model with reversible transitions are only feasible if one assumes that some of the transitions are Markov [Kang and Lagakos (2007)] or that the trajectories follow the most parsimonious path between observed states [Aralis (2016)]. In contrast, the latent CTMC modeling approach has no trouble computing transition probabilities in a wide variety of models, given that it is based on an underlying standard CTMC model. Ultimately, the latent CTMC approach offers both flexibility in terms of sojourn times and broad applicability to a variety of settings, including but not limited to the active surveillance context. 
In conclusion, our harnessing of the latent CTMC approach allows us to compare cohorts with diverse surveillance schema. The power of our approach is that it enables us to coherently use discretely observed data to investigate an underlying process that occurs in continuous time. This has widespread application in cancer research, including the study of disease recurrence following primary treatment. Using this approach, we find that perceptions of cross-cohort differences may be revealed to be artifactual, and real differences in the underlying disease progression process may be different from those assessed on the basis of empirical data.

\section{SUPPLEMENTARY MATERIAL}

Supplement A: Maximum likelihood estimation (DOI: 10.1214/17AOAS1130SUPPA; .pdf). Description of the expectation-maximization algorithm used to estimate model parameters.

Supplement B: Sample description (DOI: 10.1214/17-AOAS1130SUPPB; .pdf). Description of the four active surveillance cohorts after exclusions.

Supplement C: Parameter estimates (DOI: 10.1214/17-AOAS1130SUPPC; .pdf). Model parameter estimates assuming 100\% biopsy sensitivity and specificity.

Supplement D: Model selection (DOI: 10.1214/17-AOAS1130SUPPD; .pdf). BIC model selection for the four active surveillance cohorts, assuming $60 \%, 75 \%$, and $90 \%$ sensitivity and $100 \%$ specifcity.

Supplement E: Additional figures (DOI: 10.1214/17-AOAS1130SUPPE; .pdf). Sensitivity analysis for estimates of upgrading probabilities.

\section{REFERENCES}

Andersen, P. K. and Keiding, N. (2002). Multi-state models for event history analysis. Stat. Methods Med. Res. 11 91-115.

ARALIS, H. J. (2016). Modeling multistate processes with back transitions: Statistical challenges and applications. Ph.D. thesis, UCLA.

Baum, L. E., Petrie, T., Soules, G. and Weiss, N. (1970). A maximization technique occurring in the statistical analysis of probabilistic functions of Markov chains. Ann. Math. Stat. 41 164171.

Bladt, M. and Sorensen, M. (2005). Statistical inference for discretely observed Markov jump processes. J. R. Stat. Soc., Ser. B Stat. Methodol. 67 395-410.

Coley, R. Y., Zeger, S. L., Mamawala, M., Pienta, K. J. and Carter, H. B. (2016). Prediction of the pathologic Gleason score to inform a personalized management program for prostate cancer. Eur. Urol. 72 135-141.

CUMANI, A. (1982). On the canonical representation of homogeneous Markov processes modelling failure-time distributions. Microelectron. Reliab. 22 583-602.

Dall'Era, M. A. (2015). Patient and disease factors affecting the choice and adherence to active surveillance. Curr. Opin. Neurol. 25 272-276. 
Donnelly, C., McFetridge, L. M., Marshall, A. H. and Mitchell, H. J. (2017). A twostage approach to the joint analysis of longitudinal and survival data utilising the Coxian phasetype distribution. Stat. Methods Med. Res. To appear. PMID: 28633604.

Foucher, Y., Giral, M., SOUlillou, J.-P. and DAURES, J.-P. (2007). A semi-Markov model for multistate and interval-censored data with multiple terminal events. Application in renal transplantation. Stat. Med. 26 5381-5393.

FRYDMAN, H. and SZAREK, M. (2009). Nonparametric estimation in a Markov "illness-death" process from interval censored observations with missing intermediate transition status. Biometrics $65143-151$.

Gignac, G. A., Morris, M. J., Heller, G., Schwartz, L. H. and Scher, H. I. (2008). Assessing outcomes in prostate cancer clinical trials: A twenty-first century tower of Babel. Cancer 113 966-974.

Gilbert, P. and VARADHAN, R. (2012). numDeriv: Accurate numerical derivatives. R package version 2012.9-1.

GRÜGER, J., KAY, R. and SCHUMACHER, M. (1991). The validity of inferences based on incomplete observations in disease state models. Biometrics 47 595-605.

HuANG, X. and Wolfe, R. A. (2002). A frailty model for informative censoring. Biometrics $\mathbf{5 8}$ $510-520$.

Hudgens, M. G., SAtten, G. A. and Longini, I. M. (2001). Nonparametric maximum likelihood estimation for competing risks survival data subject to interval censoring and truncation. Biometrics $\mathbf{5 7} 74-80$.

Humphrey, P. A. (2004). Gleason grading and prognostic factors in carcinoma of the prostate. Mod. Pathol. 17 292-306.

Inoue, L. Y. T., Trock, B. J., Partin, A. W., CARter, H. B. and Etzioni, R. (2014). Modeling grade progression in an active surveillance study. Stat. Med. 33 930-939.

Jackson, C. H., Sharples, L. D., Thompson, S. G. and Duffy, S. W. (2003). Multistate Markov models for disease progression with classification error. J. R. Stat. Soc., Ser. D Stat. 52 193-209.

KANG, M. and LAgakos, S. W. (2007). Statistical methods for panel data from a semi-Markov process, with application to HPV. Biostatistics 8 252-264.

Klotz, L., Vesprini, D., Sethukavalan, P., Jethava, V., Zhang, L., Jain, S., Yamamoto, T., Mamedov, A. and Loblaw, A. (2015). Long-term follow-up of a large active surveillance cohort of patients with prostate cancer. J. Clin. Oncol. 33 272-277.

LANGE, J. M. and Minin, V. N. (2013). Fitting and interpreting continuous-time latent Markov models for panel data. Stat. Med. 32 4581-4595.

Lange, J. M., Hubbard, R. A., Inoue, L. Y. T. and Minin, V. N. (2015). A joint model for multistate disease processes and random informative observation times, with applications to electronic medical records data. Biometrics 71 90-101. MR3335353

Lange, J. M., Gulati, R., Leonardson, A. S., Lin, D. W., Newcomb, L. F., Trock, B. J., Carter, H. B., Cooperberg, M. R., Cowan, J. E., Klotz, L. H. and ETZIONI, R. (2018). Supplement to "Estimating and comparing cancer progression risks under varying surveillance protocols." DOI:10.1214/17-AOAS1130SUPPA, DOI:10.1214/17AOAS1130SUPPB, DOI:10.1214/17-AOAS1130SUPPC, DOI:10.1214/17-AOAS1130SUPPD, DOI:10.1214/17-AOAS1130SUPPE.

MANDEL, M. (2010). Estimating disease progression using panel data. Biostatistics 11 304-316.

MAO, L., LIN, D.-Y. and ZENG, D. (2017). Semiparametric regression analysis of interval-censored competing risks data. Biometrics $\mathbf{7 3} 857-865$.

Marshall, G. and Jones, R. H. (1995). Multi-state models and diabetic retinopathy. Stat. Med. 14 1975-1983.

Moler, C. and LOAN, C. V. (2003). Nineteen dubious ways to compute the exponential of a matrix, twenty-five years later. SIAM Rev. 45 801-836. 
NAROD, S. A. and RAKOVITCH, E. (2014). A comparison of the risks of in-breast recurrence after a diagnosis of DCIS or early invasive breast cancer. Curr. Oncol. 21 119-124.

Newcomb, L. F., Thompson Jr., I. M., Boyer, H. D., Brooks, J. D., Carroll, P. R., Cooperberg, M. R., Dash, A., Ellis, W. J., Fazli, L., Feng, Z., Martin, E., Kunju, P., Lance, R. S., Mckenney, J. K., Meng, M. V., Marlo, M., Sanda, M. G., Simko, J., So, A., Tretiakova, M. S., Troyer, D. A., True, L. D., Vakar-Lopez, F., Virgin, J., Wagner, A. A., Wei, J. T., Nelson, P. S. and Lin, D. W. for the Canary Prostate ACTIVE SURVEILLANCE STUDY INVESTIGATORS (2016). Outcomes of active surveillance for the management of clinically localized prostate cancer in the prospective, multi-institutional Canary PASS cohort. J. Urol. 195 206-221.

Palisaar, J. R., Noldus, J., Löppenberg, B., Von Bodman, C., Sommerer, F. and EgGERT, T. (2012). Comprehensive report on prostate cancer misclassification by 16 currently used low-risk and active surveillance criteria. BJU Int. 110.

PENSON, D. F. (2012). Factors influencing patients' acceptance and adherence to active surveillance. J. Natl. Cancer Inst. Monogr. 45 207-212.

PINSKY, P., PARNES, H. and FORD, L. (2008). Estimating rates of true high-grade disease in the Prostate Cancer Prevention Trial. Cancer Prev. Res. 1 182-186.

Popiolek, M., Rider, J. R., ANdrén, O., Andersson, S.-O., Holmberg, L., AdAmi, H.-O. and JohANSSON, J.-E. (2013). Natural history of early, localized prostate cancer: A final report from three decades of follow-up. Eur. Urol. 63 428-435.

Ross, A. E., Loeb, S., Landis, P., Partin, A. W., Epstein, J. I., Kettermann, A., Feng, Z., CARTER, H. B. and Walsh, P. C. (2010). Prostate-specific antigen kinetics during follow-up are an unreliable trigger for intervention in a prostate cancer surveillance program. J. Clin. Oncol. 28 2810-2816.

Rouanet, A., Joly, P., Dartigues, J.-F., Proust-Lima, C. and Jacqmin-Gadda, H. (2016). Joint latent class model for longitudinal data and interval-censored semi-competing events: Application to dementia. Biometrics 72 1123-1135.

SRIDhaRA, R., MANDREKAR, S. J. and DodD, L. E. (2013). Missing data and measurement variability in assessing progression-free survival endpoint in randomized clinical trials. Clin. Cancer Res. 19 2613-2620.

Steele, R. J. and RAfTERY, A. E. (2010). Performance of Bayesian model selection criteria for Gaussian mixture models. In Frontiers of Statistical Decision Making and Bayesian Analysis (M.-H. Chen, P. Muller, D. Sun, K. Ye and D. K. Dey, eds.) 113-130. Springer, New York.

Stephenson, A. J., Kattan, M. W., Eastham, J. A., Dotan, Z. A., Bianco, F. J., Lilja, H. and SCARDINO, P. T. (2006). Defining biochemical recurrence of prostate cancer after radical prostatectomy: A proposal for a standardized definition. J. Clin. Oncol. 24 3973-3978.

Titman, A. C. and Sharples, L. D. (2010). Semi-Markov models with phase-type sojourn distributions. Biometrics 66 742-752.

Tosoian, J. J., Mamawala, M., Epstein, J. I., Landis, P., Wolf, S., Trock, B. J. and CARTER, H. B. (2015). Intermediate and longer-term outcomes from a prospective activesurveillance program for favorable-risk prostate cancer. J. Clin. Oncol. 33 3379-3385.

Tosoian, J. J., CARTER, H. B., LePor, A. and Loeb, S. (2016). Active surveillance for prostate cancer: Contemporary state of practice. Nat. Rev. Urol. 116 1477-1490.

Welty, C. J., Cowan, J. E., Nguyen, H., Shinohara, K., Perez, N., Greene, K. L., Chan, J. M., Meng, M. V., Simko, J. P., Cooperberg, M. R. and Carroll, P. R. (2015). Extended followup and risk factors for disease reclassification in a large active surveillance cohort for localized prostate cancer. J. Urol. 193 807-811.

Zeng, L., Cook, R. J., Wen, L. and Boruvka, A. (2015). Bias in progression-free survival analysis due to intermittent assessment of progression. Stat. Med. 34 3181-3193. 
J. M. LANGE

R. GULATI

A. S. LEONARDSON

L. F. NEWCOMB

R. ETZIONI

FRED HutCHINSON CANCER

REESARCH CENTER

1100 FAIRVIEW AVENUE NORTH

SEATTLE, WASHINGTON 98109-1024

USA

E-MAIL: jlange@fredhutch.org rgulati@fredhutch.org aleonard@fredhutch.org lnewcomb@fredhutch.org retzioni@fredhutch.org

B. J. TROCK

H. B. CARTER

THE JAMES BUCHANAN UROLOGICAL INSTITUTE JOHNS HOPKINS MEDICAL INSTITUTIONS 600 NORTH WOLFE STREET

BALTIMORE, MARYLAND 21287

USA

E-MAIL: btrock@jhmi.edu carter.ballentine3@gmail.com
D. W. LIN

DEPARTMENT OF UROLOGY

UNIVERSITY OF WASHINGTON

1959 NE PACIFIC STREET

SEATTLE, WASHINGTON 98195

USA

E-MAIL: dlin@uw.edu
P. R. CARROLL

M. R. COOPERBERG

J. E. COWAN

DEPARTMENT OF UROLOGY

UNIVERSITY OF CALIFORNIA,

SAN FRANCISCO

400 PARNASSUS AVENUE

SAN FRANCISCO, CALIFORNIA 94143

USA

E-MAIL: Matthew.Cooperberg@ucsf.edu Janet.Cowan@ucsf.edu

L. H. KLOTZ

DIVISION OF UROLOGY

UNIVERSITY OF TORONTO

2075 BAYVIEW AVENUE

TORONTO, ONTARIO M4N 3M5

CANADA

E-MAIL: Laurence.Klotz@sunnybrook.ca 